\title{
REGARD SUR L'ÉVOLUTION HISTORIQUE DU COURRIER DES LECTEURS DANS LA PRESSE
}

\author{
Marie Widart ${ }^{1}$ et Frédéric Antoine ${ }^{2}$
}

\section{Introduction}

Les sciences de la communication ont bien longtemps ignoré dans leur étude des médias le pôle plus discret mais pourtant essentiel des récepteurs. En effet, le journal, fruit d'une écriture rédactionnelle, n'a d'autre but que de diffuser de l'information dans la sphère publique et d'échoir ainsi dans les mains de lecteurs. Il est dès lors nécessaire de prendre en compte les "consommateurs" du produit médiatique. Or, comme le soulignent Bianchi et Bourgeois, il serait simpliste de penser que "ces récepteurs sont a priori privés d' initiatives et de stratégies, incapables de créer du sens (se bornant à enregistrer, à déformer ou à rejeter, celui qu'a fabriqué l'émetteur.)"3. Au contraire, les récepteurs tiennent aujourd'hui un rôle actif dans la circularité des échanges entre médias et usagers. Trop longtemps

1 Chercheur FNRS (Fonds National de la Recherche Scientifique) au Département de communication de l'Université catholique de Louvain.

2 Professeur au Département de communication de l'Université catholique de Louvain.

3 J. Bianchi et H. Bourgeois, Les médias côté public, Paris, 1992, pp. 34-35.

Recherches en communication, $\mathrm{n}^{\circ} 21$ (2004). 
considérés comme simples consommateurs médiatiques, ils disposent à présent de divers espaces d'expression au sein des médias. L'un d'eux s'apparente aux rubriques du courrier des lecteurs, publiées dans certains journaux et périodiques. Ainsi, et toutes proportions gardées, peut-on estimer que le courrier des lecteurs constitue une des formes "d'agora moderne", reconnu par les usagers comme un lieu de don de la parole privilégié. À ce titre, il est un accès intéressant à l'opinion des lecteurs ${ }^{1}$ et justifie dès lors la présente étude.

Quelle place les différents journaux et périodiques confèrent-ils réellement à l'expression de leurs lecteurs ? Qui sont ces personnes désireuses de s'exprimer par le biais du courrier des lecteurs? Quelles sont les motivations profondes de la prise de parole de l'usager? Quel rôle ce dernier entend-il jouer dans sa relation aux médias ? Quand les premiers signes de l'évolution de la relation usagers/médias sont-ils apparu ? Et, au bout du compte, peut-on parler d'une véritable "coconstruction" entre usagers et médias? Autant de questions auxquelles le présent article tentera d'apporter de modestes amorces de pistes de réponse en ébauchant l'esquisse d'un tableau général de l'évolution du courrier des lecteurs au sein de la presse, en Belgique francophone pour l'essentiel.

Pour établir ce bref panorama, nous avons choisi d'analyser uniquement la presse nationale, sélectionnant pour notre étude cinq quotidiens et un hebdomadaire : La Libre Belgique (Le Patriote de 1883 à 1914, puis La Libre Belgique à partir de 1918), Le Soir (créé en 1887), Le Peuple (publié à partir de 1886), La Nation Belge (19181955), Le XX $X^{e}$ Siècle (1897-1939) et Pourquoi Pas ? (1910-1988). Les divers quotidiens traversent une large période et couvrent diverses tendances idéologiques. Quant au choix du Pourquoi Pas?, il s'imposait au vu de la politique d'ouverture à ses "usagers" pratiquée par cet hebdomadaire tout au long de son histoire. Celui-ci est en effet le seul à publier sans interruption une imposante rubrique de courrier des lecteurs depuis sa création en 1910 jusqu'à sa disparition en $1988^{2}$. Pour chacun de ces journaux, nous avons procédé par coups de sonde réguliers en sélectionnant par an une même semaine de l'année ${ }^{3}$,

1 Notons cependant qu'il nous donne à lire uniquement le point de vue de ceux qui désirent s'exprimer et ne reflète donc pas l'avis de l'ensemble de la population.

2 Proportionnellement, Pourquoi Pas? publie ainsi trois fois plus de courriers que $L a$ Libre Belgique ou Le Soir, sept fois plus que Le Peuple ou La Nation Belge et près de trente fois plus que $L e X X^{e}$ Siècle, dont la production demeure fort marginale.

3 Nous avons choisi cette semaine, de façon aléatoire, autour du 29 novembre. 
depuis leur création jusqu'en 1990, afin de tenter de saisir quelques indications synoptiques sur les évolutions inhérentes au courrier des lecteurs de la presse belge depuis la fin du $\mathrm{XIX}^{\mathrm{e}}$ siècle.

\section{L'évolution chronologique : plusieurs modèles de participation des lecteurs}

Pour une part au moins, il nous semble que les débuts des rapports épistolaires entre lecteurs de journaux et producteurs d'organe de presse doivent se situer dans un registre aujourd'hui bien oublié, ne concernant pas tant le domaine de la rétroaction de l'usager face à un contenu publié que celui de la fourniture d'informations à l'organe de presse.

Il est ainsi significatif que plusieurs titres d'organes de presse comportaient jadis le mot "Courrier". Ce terme figure toujours dans l'intitulé de certaines éditions de quotidiens régionaux belges. Indépendamment de ce fait, une lecture transversale d'anciens titres de la presse régionale française et en particulier une analyse de quelques très anciens numéros du journal Le Progrès de Lyon nous a confirmé dans l'impression que "le courrier" joua jadis dans la presse un rôle considérable. Au milieu du $\mathrm{XIX}^{\mathrm{e}}$ siècle, au moment où naît ce quotidien régional français, les lettres qui y sont publiées constituent en effet souvent des éléments informationnels d'actualité que le journal ne peut livrer à ses lecteurs que grâce au concours de ces "informateurs" très particuliers. Dans une partie des cas rencontrés, ces fournisseurs de nouvelles peuvent être considérés comme des “correspondants" du journal au sens classique du terme. C'est-à-dire des personnes à qui le quotidien a confié la tâche de recenser des événements auxquels ils ont assisté. Leurs textes ne répondent toutefois à aucune norme classique d'écriture journalistique : ils sont généralement publiés à l'état brut, sous forme de missives. Ces "correspondances" dont le journal se targue de la qualité ${ }^{2}$ manifestent

1 Que l'on songe par exemple au Courrier de Versailles à Paris, au Courrier de Provence ou à des titres étrangers comme le Globe and Mail ou le Daily Mail... Et, dans leur Histoire de la presse après 1945, Archambault et Lemoine recensent encore plus de trente titres de presse française comprenant le mot "courrier".

2 "A nos abonnés. Le Progrès n'existe que depuis trois ans, et personne ne contestera qu'il a déjà su se faire une place dans le journalisme libéral. "Il n'y a pas en France de journal mieux rédigé que le Progrès", disait La Presse du 10 décembre 1862. 
une incontestable présence d'un "courrier" très particulier dans son rédactionnel. Dans le cas du Progrès, au milieu du $\mathrm{XIX}^{\mathrm{e}}$ siècle, toutes les nouvelles que ce quotidien régional diffuse en provenance de la capitale et de la vie politique qui s'y déroule passent par ce type de communication.

D'autres lettres trouvées lors d'une lecture flottante de numéros anciens de ce journal lyonnais témoignent d'une intervention directe de lecteurs, qui n'ont pas le titre de correspondant officiel du journal, mais se plaisent à lui transmettre des nouvelles ou des informations ${ }^{1}$ que l'organe de presse reproduit à titre de nouvelle. Dans d'autres cas, on trouve publiés des extraits de lettres dont on ne sait s'il s'agit de "correspondances" officielles commandées par le journal ou de la publication de correspondances reçues par le journal sans qu'il y ait eu sollicitation ${ }^{2}$.

Il semble en tout cas que, au cours du XIX ${ }^{\mathrm{e}}$ siècle, une partie de la presse, encore semi-professionnelle, permit à ses lecteurs de ne pas se contenter d'un rôle passif, mais de participer dans une certaine mesure à l'alimentation du journal en matière première. Et ceci sans que la forme ou le fond de cette information ne soient retravaillés.

Deux jours après, Le Temps rangeait Le Progrès à côté du Phare de la Loire et de la Gironde parmi les rares feuilles de province "qui font autorité". (...) Le premier devoir de tout journal, c'est d'être un organe de publicité et par conséquent de donner les faits qui intéressent le public le plus RAPIDEMENT et le plus COMPLETEMENT possible. (...) Combien de journaux de province acceptent pour correspondants des individus sans notoriété politique ou littéraire, qui n'écrivent cette chronique au jour le jour des nouvelles parisiennes que faute de savoir écrire des article! Nous avons confié cette partie de la rédaction, souverainement importante selon nous, à des écrivains distingués et appelés par leur mérite même et par leur rôle à voir de près les détails intimes de la vie publique et pour ainsi dire le dessous des cartes. (...)" (04/01/1863).

1 Ainsi, par exemple, ce texte sans titre du 3 janvier 1863 : "M Lafond nous avait déjà adressé une première lettre sur la liberté de courtage. Nous en recevons une seconde aujourd'hui qui aura d'autant plus d'intérêt que nos lecteurs liront aujourd'hui l'arrêt de la cour de Lyon sur cette importante question.". Cette introduction, signée A De Rolland, est suivie d'un texte commençant de la sorte : "A monsieur le directeur du Progrès.

Il y a quelques jours, le Salut public publiait un article traitant encore (...)".

Ainsi encore ce texte intitulé "Correspondance universelle", publié le 6 janvier 1863, dans lequel un inventeur présente un système de télégraphe à installer dans chaque maison...

2 Ainsi, par exemple : ce texte intitulé "Correspondance parisienne" signé Emile Gaboriau et publié le $1^{\text {er }}$ janvier 1863. Il est rédigé comme suit: "Paris, 30 décembre. Monsieur, j'entends dire, comme cela, que le temps n'est pas aux Revues. C'est tout à fait mon sentiment.(...)" 
Cette situation était encouragée par la configuration formelle de type épistolaire utilisée dans une partie importante des textes publiés.

À la fin du XIX ${ }^{\mathrm{e}}$ siècle, on retrouve ponctuellement la même situation dans certains des organes de presse belges francophones sur lesquels nous avons posé un regard.

Les journaux qui commencent alors à paraître publient régulièrement des courriers de lecteurs. Ces courriers demeurent toutefois épisodiques et n'apparaissent pas au sein d'une rubrique précise. Ils sont souvent insérés entre deux articles, sans titre ni chapeau. Le Patriote publie ainsi un grand nombre de courriers (en moyenne, trois fois plus que les autres journaux). Parmi eux, le quotidien fait place à quelques courriers purement informatifs. Ils sont rédigés par des lecteurs qui n'écrivent pas pour donner leur avis sur un sujet de l'actualité mais uniquement pour informer le journal de la création d'un nouveau tronçon de chemin de fer, des activités du Roi ou de la situation politique à l'étranger. Ces courriers, quelque peu atypiques, participent dès lors à une véritable co-construction du journal mais leur importance s'estompera dès le début $\mathrm{du} \mathrm{XX}^{\mathrm{e}}$ siècle pour disparaître complètement au lendemain de la Première Guerre mondiale.

\section{L'ère industrielle de la presse : un lecteur consommateur}

Lorsque s'installe le $\mathrm{XX}$ e siècle et que la presse prend une configuration plus industrielle et professionnelle, les rôles semblent se figer davantage : l'information devient l'unique affaire des journalistes et de ceux que ceux-ci choisissent pour être leurs interlocuteurs. Les lecteurs sont invités à se nourrir des contenus diffusés, et éventuellement à réagir à leur lecture. Mais ils ne sont plus directement considérés comme associés à l'élaboration du contenu informationnel du journal.

Tout au plus leur permet-on parfois d'alimenter les contenus du journal en abordant, dans une lettre publiée, une réaction vis-à-vis de problèmes de proximité vécus par le lecteur, et que le journal n'aurait peut-être pas de lui-même monté en épingle : le mauvais entretien des trottoirs, le retard des trains, les exagérations du fisc...

Cette tendance ira en s'accentuant tout au long de la première moitié de ce siècle, et même un peu au-delà.

Durant l'entre-deux-guerres, dans les journaux belges, les courriers des lecteurs se font encore plus rares et s'insèrent 
uniquement dans des rubriques de petites informations variées comme "La Journée" dans La Libre Belgique ou "D'un jour à l'autre" dans Le Peuple. Au sein de ces rubriques, ils prennent un caractère quelque peu anecdotique et toujours très irrégulier. En 1939 cependant, les journaux ouvrent assez largement leurs colonnes à l'expression de leurs lecteurs à propos de la guerre. La Nation Belge crée pour l'occasion une rubrique intitulée "Courrier du soldat" où de nombreux mobilisés décrivent leur difficile situation. Thème fédérateur, le contexte particulier de la guerre délie donc les plumes et offre surtout une véritable tribune aux lecteurs à la veille du conflit.

L'immédiat après-guerre connaît un mouvement inverse : cette période est marquée par une absence quasi généralisée du courrier des lecteurs (à l'exception du Pourquoi Pas ? qui publie des courriers sans interruption). La presse, qui doit se reconstruire au lendemain du conflit, présente un profil essentiellement informationnel et ne s'intéresse guère au point de vue des lecteurs. Seule une rubrique de conseils en réponse à des interrogations des lecteurs assure un lien ténu avec ceux-ci. L'absence de courrier des lecteurs traduit alors une certaine façon d'envisager le rôle de la presse et son rapport au lecteur. Celui-ci ne se voit donc pas reconnu une place d'interlocuteur mais est considéré comme un simple "réceptacle d'informations".

\section{Presse et post-modernité : le retour du lecteur}

Un net tournant s'opère au cours des années 1970. Les rubriques du courrier des lecteurs se multiplient en effet dans presque tous les journaux. Elles paraissent à intervalles réguliers, toujours au même endroit dans le journal, le plus souvent en page "Débat et Opinions". Elles portent un titre et s'amplifient considérablement pour accueillir parfois plusieurs courriers par jour. Certes, les appellations de ces rubriques varient (parfois au sein d'un même journal) de "Nos lecteurs et..." dans La Libre Belgique (régulière à partir de 1974) à "Mon mot à dire" pour Le Peuple (dès 1979), en passant par les trois titres du Soir "Le Forum de l'opinion" (1972-1982), "Quinze lignes pour le dire" (1983-1989) et "Le Courrier des lecteurs" (à partir de 1990) mais derrière chacun de ces intitulés se développe toujours une rubrique systématique, ouverte à la prise de parole des lecteurs. Par ailleurs, le nombre de courriers publiés dans ces nouvelles rubriques 
augmente lui aussi considérablement. Parmi les courriers recensés dans notre étude, Le Peuple en publie un tiers à partir de 1979, La Libre Belgique la moitié à partir de 1974 et Le Soir les trois-quarts depuis 1972. Les premiers signes d'une véritable attention à l'expression des lecteurs se retrouvent dès lors dans cette phase, sorte de "décollage" des rubriques du courrier des lecteurs dans les années 1970.

\section{Médiation-Opinion-Action}

On a coutume de considérer que les médias actuels sont revêtus par leurs usagers de rôles d'intercesseurs ou de médiateurs peu connus jusqu'ici. La littérature consacrée à l'évolution des modèles télévisuels fait la part belle à l'avènement de ces médias "médiateurs" ou "acteurs" chargés par le public d'intervenir afin de pallier les dysfonctionnements sociaux. Un regard rétrospectif dans le courrier des lecteurs de la presse démontre que cette attente du public à l'égard des médias n'est pas à proprement parler une chose neuve et liée aux médias audiovisuels.

À la fin du XIX $\mathrm{X}^{\mathrm{e}}$ siècle surtout mais encore au début $\mathrm{du} \mathrm{XX}^{\mathrm{e}}$ siècle, nombreux sont même les lecteurs qui demandent au journal de jouer un rôle d'intercesseur par rapport aux autorités en charge de leur problème. Ainsi, ces nombreuses prières de lecteurs qui considèrent leur quotidien comme l'ultime recours face à une situation inextricable et sollicitent clairement son intervention.

"Nous espérons que $M$. Liehaert nous fera rendre justice et nous comptons sur le "XX ${ }^{\mathrm{e}}$ Siècle" pour lui faire connaître nos légitimes doléances."1

"Je viens vous prier d'entreprendre une campagne contre cet abus scandaleux, et, à cet effet, je vous demande, pour ma lettre, l'hospitalité de vos colonnes."2

"Nous venons vous prier d'attirer sur nos services la bienveillante attention de M. le Ministre."3

"Ne pourriez-vous signaler à qui de droit, la situation qui nous est faite au point de vue du service postal ?"4

1 Le XX' Siècle, le 28/11/1905, n 332, p. 1.

2 La Libre Belgique, le 26/11/1895, $n^{\circ} 330$, p. 1.

3 La Libre Belgique, le 30/11/1905, $\mathrm{n}^{\circ} 334$, p. 2.

4 La Libre Belgique, le 03/12/1905, n ${ }^{\circ} 337$, p. 2. 
"L'intérêt que vous portez pour le bien-être général nous étant connu, nous espérons que vous voudrez bien appuyer notre demande dans votre honorable journal."1

À ce titre, Pourquoi Pas? entretient un contact tout à fait particulier avec ses lecteurs. La majorité des lecteurs qui écrivent de l'hebdomadaire le tutoie et s'adresse à lui comme à un ami. Beaucoup font son éloge, l'assurent de leur fidélité ou lui servent du "Cher Pourquoi Pas ?". Tout cela témoigne du lien très fort (et unique) que le périodique a su établir avec ses lecteurs. Interlocuteur privilégié, il est un peu un ami à qui l'on confie plaintes et espoirs. Bien plus, il est un porte-parole et un intermédiaire auquel on prête un pouvoir d'action. Confiants en leur hebdomadaire, certains lecteurs-écrivants n'hésitent donc pas à lui demander d'intervenir en leur faveur.

"J'habite avenue Albert. Albert quoi ? C"est ce que je me demande tous les jours. (...) Si c'est pour perpétuer le souvenir d'un roi qui n'est pas le premier venu (...), qu'on dise donc : "Avenue Albert Ier". Quelle autre allure cela vous aurait ! (..) Si Pourquoi Pas?" pouvait faire quelque chose pour cela, il serait comblé de remerciements..."2

Il semble donc que la rubrique du courrier des lecteurs, au-delà d'un simple espace cathartique ouvert à l'opinion, se voit confier un rôle plus actif par le lecteur, en sa qualité d'intercesseur.

Une fonction plus globale du courrier des lecteurs, découlant de celle qui précède, est aussi de permettre aux citoyens d'exprimer une plainte, une opinion. La communication de points de vue a toujours constitué une des grandes catégories de contenu des rubriques de courriers de lecteurs, et on peut même considérer que ces espaces ont été un des lieux-refuges de l'expression d'opinions alors que celles-ci devenaient de moins en moins nombreuses dans les autres pages des journaux. Derrière ces plaintes et ces avis, on retrouve une constante : celle de la critique.

Ainsi, ce socialiste désabusé qui clame dans la presse son dégoût de la gauche.

“Ancien socialiste (...) puis-je obtenir le droit de crier ce que j'ai sur le cour à haute voix (...). [Notre société] est pourrie à cause des parlementaires qui, une fois élus, se fichent

1 La Libre Belgique, le 29/11/1885, n³33, p. 2.

2 A.C., dans Pourquoi Pas ?, le 28/11/1930, n 852, p. 2542. 
éperdument de leurs électeurs. Ce parti (...) dont le président vient à la TV raconter ses balivernes aux imbéciles qui se laissent berner chaque fois. Je souhaite à ce parti la plus grande défaite électorale possible aux prochaines élections (...)."1

De même, ce courrier virulent d'un lecteur qui se plaint de la rubrique de La Libre Belgique dont l'unique vocation semble être, selon lui, d'accueillir les propos acerbes de râleurs... dont il fait luimême partie.

"Il y a un mois, j'ai souligné le caractère agaçant et peu chrétien de cette rubrique regroupant tous ceux qui "râlent". À ma lettre, je n'ai pas même reçu un acte de réception mais la valse continue (...). Mais que nous importe ce que pensent ces gens?"2

Et en effet, les lecteurs qui écrivent aiment à se plaindre dans les colonnes d'un journal, sorte de forum où se mêlent à l'envi doléances et griefs. Rien d'étonnant dès lors à ce que le ton employé par ces lecteurs soit le plus souvent emporté ou amer, tels ces commentaires révoltés de deux lectrices.

"Outrée, oui, je suis outrée, M. Coens, de l'annonce que j'ai entendue ce matin à la radio. Quoi! Vous osez décorer un criminel de guerre, un nazi, condamné deux fois à mort par le gouvernement belge ! (...) À vous dégoûter d'être belge et patriote."3

"Les Belges ont investi au Congo, encouragés par l'État durant la crise économique, afin de contribuer au développement du pays, prolongement de notre patrie ! L'État a palpé ainsi des taxes de toute nature! Mais après avoir raté son expérience unique au monde (...) la Mère-Patrie nous a abandonnés à notre triste sort. Il est malheureux de devoir dépendre de son enfant à 65 ans, après une longue vie de travail dans un climat éprouvant. (...) J'ai appris à haïr la Belgique !"4

Le journal se fait alors confident pour accueillir les préoccupations des lecteurs sur des domaines extrêmement variés. Qu'il s'agisse

1 M. BuRY, dans Le Soir, le 01-02/12/1990, n 280, p. 2.

2 La Libre Belgique, le 29/04/1982, ${ }^{\circ} 119$, p. 2.

3 R. Eelbo, dans Le Soir, le 28/11/1985, n 277, p. 13.

4 D.R., dans Pourquoi Pas?, le 25/11/1965, n² 2452, p. 167-168. 
de réfections de la route, de la situation des pensionnés, des désagréments des transports en commun ou de la politique du gouvernement, tous se mobilisent pour dénoncer "l'inacceptable".

Au-delà de son oreille compatissante, le journal est aussi un produit destiné à la sphère publique. Les lecteurs-écrivants s'adressent à lui en cette qualité et espèrent voir leur lettre publiée. Ainsi, non contents de l'élever au rang de confident, ils investissent le journal d'une fonction de porte-voix, bien loin des secrets d'une confession. Car qui mieux que la presse peut interpeller un large public ? Par la publication de leur lettre, les lecteurs attendent donc du journal qu'il diffuse leur point de vue et rallie peut-être ainsi d'autres lecteurs à leur cause.

Par ailleurs, une part nettement moins importante des écrivants prennent la plume dans un autre but : celui de suggérer au journal (et par lui, à la collectivité) des solutions pour résoudre des problèmes soulevés par l'actualité ou éviter qu'une situation ne dégénère. Ces discours, tant préventifs que curatifs, se développent essentiellement en période de crise : marasme économique, instabilité gouvernementale, mouvements de grève, conflit communautaire, décolonisation, développement de la criminalité organisée... Autant d'événements qui, hormis les critiques qu'ils suscitent, donnent également l'occasion à certains d'exposer leurs pistes de réponse. Là encore, le journal est utilisé comme amplificateur, seul capable de relayer des suggestions auprès du plus grand nombre.

"Comme la plupart des habitants de ce pays, je suis consterné par les trop nombreux crimes commis ces dernières années, et restés impunis. Il semble assuré que nos corps de gendarmerie et de polices (...) ne sont pas à la hauteur de leur tâche. Manque de motivations ?... armement insuffisant ?... manque d'argent ?... Peut-être, tout simplement, manque d'idées! (...) Lorsque l'on sait que c'est presque toujours une VW GTI que les bandits utilisent, pourquoi ne voit-on pas : (...) Recherche systématique de toutes les caches possibles de voiture volées (...) Appels fréquents pour aide de la population.".

"Je comprends la réaction hollandaise à la réception des eauxpoubelles qu'ils reçoivent... (...) Et pourquoi donc l'État, en connaissance de cause, n'agit-il pas au plus vite en faisant des emprunts pour une raison aussi impérieuse..."2

1 J.T., dans Pourquoi Pas ?, le 27/101/1985, nº 3496, p. 208.

2 VANDENBERG, dans Le Soir; le 29/11/1980, n² 280, p. 5. 
Enfin, la rubrique du courrier des lecteurs accueille également les réponses de lecteurs voulant réagir aux articles publiés par leur journal ou aux propos tenus par d'autres lecteurs dans cette même rubrique. Cette démarche relève alors d'un processus de feedback.

En effet, les lecteurs interviennent parfois dans la rubrique du courrier pour répondre à un autre correspondant ou rectifier des propos tenus par la rédaction. Il s'agit alors de donner un retour à leur quotidien ou leur hebdomadaire. Regards des lecteurs sur la presse, ces feedbacks mettent en lumière tant la place qu'ils désirent prendre dans le processus d'élaboration de leur journal que le type de relations que celui-ci entend établir avec le pôle du récepteur. Mais il apparaît que ces feedbacks, certes présents, ne sont pas nombreux. Ils ne représentent pas plus d'un cinquième des courriers publiés. Manque d'initiative des lecteurs ou limitation voulue par les journaux ? Il n'est guère aisé de l'établir. Quoi qu'il en soit, il semble clair que les lecteurs, s'ils prennent régulièrement la plume pour écrire à leur journal, ne le font pas dans l'unique but de se positionner par rapport à celui-ci et usent finalement assez peu de leur liberté de rétroaction.

Par ailleurs, ces feedbacks peuvent exprimer la critique autant que les louanges. Les auteurs se font tantôt virulents, tantôt encourageants et les journaux ouvrent dès lors leurs colonnes ici aux reproches, là aux félicitations. Mais les divers quotidiens et hebdomadaires ne réagissent pas tous pareillement face aux critiques négatives exprimées par certains lecteurs. D'aucuns maintiennent leur point de vue ou publient les jours suivants un abondant courrier, défenseur de leur cause, tandis que d'autres accueillent sans faux-fuyants ces courriers dérangeants. On observe là les limites du processus de rétroaction car sous des dehors d'ouverture, certains journaux se montrent peu accueillants face à la critique de leurs lecteurs. Il leur est ainsi possible de ne pas publier certaines lettres, de démonter l'argumentation d'un protestataire, voire de présenter une majorité de courriers au discours laudatif sur leur rédaction et dès lors d'éluder subtilement le retour négatif de leurs lecteurs.

Ainsi, Le Soir et, dans une moindre mesure La Libre Belgique, se montrent particulièrement ouverts à la critique. Ils acceptent bien souvent les reproches et ne cherchent pas à justifier leurs propos. En témoignent ces extraits de courriers publiés à près d'un siècle d'intervalle. 
"Je lis dans le numéro hier du "Soir" un articulet relatif à l'élection qui a eu lieu dimanche dernier à Saint-Gilles. D'après cet articulet, les électeurs auraient reçu des bulletins de deux formes différentes, l'un disant qu'on pouvait voter pour deux candidats, l'autre portant qu'il n'y avait qu'un candidat à élire. Je tiens, Monsieur le directeur, à réduire à ses véritables proportions l'incident qui s'est produit et qu'on semble vouloir exploiter avec une mauvaise foi évidente."1

"Je me pose la question de savoir pour quelle raison mystérieuse votre journal s'acharne à ridiculiser l'expérience "900" que nous réalisons à Liège depuis deux ans."2

De même, La Libre Belgique laisse place à la contestation, parfois même virulente.

"Votre quotidien, habituellement pondéré a cédé à l'hystérie collective à propos du vote des Nations-Unies sur le sionisme." 3

Par contre, Le Peuple n'hésite pas à répondre directement à ses interlocuteurs ou à faire paraître, en compensation, de nombreux courriers élogieux sur son compte.

Voici, par exemple, un courrier vindicatif et menaçant auquel le journal ne manque pas de répliquer :

"Comme c'est la seconde fois que je prends votre journal en flagrant délit de mensonge, je vous requiers d'insérer ma réponse à la première page de votre prochain numéro et je laisse l'appréciation de mon conseil d'administration le soin de décider quelle suite il voudra donner à la chose. (...) Vous avez cru devoir admettre que le sieur Octors se livre à une charge à fond contre mon établissement ; or, toutes ses élucubrations ne sont basées que sur des inexactitudes flagrantes". 4

Et Le Peuple de rétorquer :

"Commençons par dire que $M$. Vimenet a tort de vouloir me séparer d'Elbers (...)"5..

1 VAn MEenen, dans Le Soir, le 28/11/1895, n 332, p. 2.

2 LAURENT, dans Le Soir, le 27/11/1985, $\mathrm{n}^{\circ} 276$, p. 23.

3 G. RENARD dans La Libre Belgique, le 26/11/1975, $n^{\circ} 330$, p. 18.

4 J. VENIMET dans Le Peuple, le 28/11/1895, n 332, p. 1.

5 Le Peuple, le 28/11/1895, n 332 , p. 1. 
Suit alors une réponse vive, voire emportée, de la rédaction qui publie cette lettre sous la menace mais maintient son point de vue.

En outre, le même jour, le quotidien présente en opposition une lettre d'un autre lecteur, congratulant cette fois le journal.

"Quoique la lecture de votre estimable journal nous soit strictement défendue, il est quand même lu par la plus grande partie de mes camarades. Nous constatons avec plaisir que souvent vous voulez bien vous occuper de nos simples, mais braves troupiers."

De même, La Nation Belge n'accorde aucune place aux critiques négatives mais publie quelques courriers aux accents approbateurs, ponctués de fréquents "tout à fait d'accord avec vous, Chère Nation Belge" 2, "dans l'excellent article que vous avez publié (...)" 3 , ou encore "C' est avec intérêt et avec satisfaction que le public suit, dans vos colonnes (...)"4.

Quant au Pourquoi Pas?, contrairement aux autres journaux et conformément à la proximité qu'il installe avec ses lecteurs, il fait la part belle à leurs commentaires, tant négatifs que positifs. Ces courriers portent ainsi la marque de la relation établie entre Pourquoi Pas? et ses lecteurs. Ceux-ci le flattent amicalement ou expriment sans détour leur critique. La règle implicite semble être à la transparence. Pourquoi Pas?, comme l'atteste ce commentaire de la rédaction et le courrier qui l'accompagne, se targue d'ailleurs d'être le seul hebdomadaire où le débat est réellement ouvert.

"Notre dernier éditorial réclamant la démission du ministre de Vleeschauwer nous a valu un courrier extraordinairement abondant. Il nous est, hélas ! tout à fait impossible d'en envisager la publication même partielle. Toutefois, nous avons décidé de publier la plus courte de ces innombrables lettres parce qu'il nous paraît qu'elle résume parfaitement toutes les autres. Nous tenons à remercier de tout cœur les centaines de lecteurs qui ont voulu, à cette occasion, nous exprimer leur approbation et nous prodiguer leurs encouragements." 5

Et le lecteur de confirmer, enthousiaste :

1 Le Peuple, le 28/11/1895, n 332, p. 1.

La Nation Belge, le 28/11/1933, $\mathrm{n}^{\circ} 332$, p. 7.

3 La Nation Belge, le 28/1 1/1933, n 332 , p. 7.

4 La Nation Belge, le 29/11/1929, n 333, p. 2.

5 Pourquoi Pas?, le 25/11/1960, n² 2191, p. 150. 
"Félicitations, et de tout cœur, pour ton article sur le "baron" de Vleeschauwer! Continue de dénoncer les abus et les scandales des politiciens de TOUS les partis. Tu es un des journaux libres de ce pays, reste-le. Ton maintien est la garantie de la santé de notre démocratie"l.

Mais Pourquoi Pas? fait également place à la critique, publiant, par exemple, ce courrier d'un pharmacien révolté.

"Suite à votre article dans "Pourquoi Pas ?", n 1112, page 2655 , je constate une fois de plus que lorsqu'il s'agit de pharmacie, ou pharmacien, votre "imbécillité" dans "ce" domaine n'a d'égale que votre insigne mauvaise foi." 2

Et Pourquoi Pas? de répliquer, non sans ironie :

"Grâce! Grâce! Assommé le samedi, nous n'avons repris connaissance que le lundi et nous demeurons épouvantés, sans un poil de sec. Ah! On ne rigole pas, dans la pharmacie de Tongres..." 3 .

Ainsi, le processus de rétroaction, dont la rubrique du courrier des lecteurs reste un espace privilégié, présente-t-il un bilan en demiteintes. Certes, les lecteurs s'expriment de temps à autre par ce biais pour renvoyer un feedback à leur journal, mais l'accueil de la presse est parfois mitigé et le nombre, somme toute fort limité, de ce type de courriers atteste peut-être aussi de la faiblesse du genre.

\section{La rubrique des courriers des lecteurs : une expression sous contrôle}

Si la rubrique du courrier des lecteurs offre l'occasion aux lecteurs d'investir leur journal, celui-ci, par le choix des lettres qu'il publie et par ses commentaires ensuite, reste bien le maître de l'espace concédé. Le lecteur se voit accorder un don de la parole mais dans un cadre fixé par le journal. La rubrique du courrier des lecteurs n'est donc pas à proprement parler un espace en friche, complètement

1 J.L., dans Pourquoi Pas?, le 25/11/1960, n 2191, p. 150.

2 Pourquoi Pas, le 29/1 1/1935, n ${ }^{\circ} 1113$, p. 2775.

3 Pourquoi Pas, le 29/11/1935, n² 1113, p. 2775. 
laissée aux mains des récepteurs. Elle demeure avant tout une rubrique parmi d'autres du quotidien ou du périodique. Elle porte dès lors son empreinte et reflète partiellement sa vision des choses. Ainsi, le choix des courriers publiés reste l'apanage du journal et sert souvent, dans une certaine mesure, sa ligne rédactionnelle. La tendance des divers journaux transparaît dès lors discrètement dans le choix des thèmes abordés, surtout à la fin du $\mathrm{XIX}^{\mathrm{e}}$ et au début du $\mathrm{XX}^{\mathrm{e}}$ siècle, époque où les couleurs politiques s'exprimaient encore sans détour dans la presse. Rien d'étonnant dès lors à ce que La Nation Belge ouvre largement ses colonnes aux courriers sur le patriotisme, que les lettres de La Libre Belgique traitent essentiellement des institutions telles que l'armée, le Roi et l'Église et que Le Peuple fasse la part belle aux revendications socialistes.

Ainsi, ce courrier paru dans La Nation Belge honore-t-il sans détour les vertus du patriotisme tandis que le suivant, issu du Peuple, glose sur la nécessité de l'action syndicale.

\begin{abstract}
"Puisse Raymond avec son petit émule bruxellois Fernand Met den Ancxi, mort comme lui pour avoir voulu défendre son pays, donner l'exemple de l'union sacrée à tous les Belges vivants encore : que les grands sachent vivre pour faire vivre une seule Belgique, les petits eux ne portaient, ne connaissaient qu'un nom "Belge" et pour ce nom ils ont su mourir. (...) je dis aux boches : "Venez j'y suis" et aux lâches je dis : "Retirez-vous"." 1
\end{abstract}

"Nos efforts doivent donc se porter surtout vers l'intensification de l'action directe des syndicats. Certains pensent que l'action parlementaire en tiendrait lieu le jour où le peuple jouirait entièrement du droit de suffrage (...). C'est un mirage décevant, car la puissance politique d'une classe ne tient pas à la possession du bulletin de vote, mais est en raison directe de son influence dans le domaine économique."2

D'autre part, les lignes idéologiques des diverses rédactions apparaissent également en filigrane au sein même de leur rubrique du courrier des lecteurs par les commentaires qui accompagnent les lettres publiées. Traces plus ou moins discrètes véhiculées par le journal, ces réflexions laissent très clairement entrevoir l'engagement et la sensibilité du quotidien ou de l'hebdomadaire et montrent, une

1 Museu, La Nation Belge, le 03/12/1920, n 338, p. 1.

2 J. Roggeman, dans Le Peuple, le 29/1 1/1905, n³33, p. 1. 
fois de plus, que la rubrique des courriers des lecteurs porte la marque du journal dans lequel elle s'insère. La Nation Belge honore dès lors tout acte de patriotisme dans ses commentaires tandis que Le Peuple introduit bon nombre de ses courriers par une diatribe sur le socialisme ou des propos nettement anticléricaux.

\begin{abstract}
"Personne ne lira sans émotion cette lettre admirable où le patriotisme fait taire la douleur paternelle et s'exprime en des accents à la fois simples et sublimes." 1

"Dans l'une de ses éditions de septembre, l'hebdomadaire paroissial "Dimanche" publiait l'avertissement suivant qui a fait bondir les tenants de la morale laïque, et notamment l'un de nos lecteurs. Voici ce texte intolérant autant qu'intolérable. (...) Le lecteur, qui nous écrit ses impressions à ce sujet, se demande si l'on veut rebrûler Jean d'Arc." 2
\end{abstract}

Ainsi, la rubrique du courrier des lecteurs porte-t-elle naturellement l'empreinte du journal dont elle est issue, au même titre que tout autre rubrique. Il serait dès lors erroné d'aborder la rubrique des courriers des lecteurs comme l'œuvre des seuls lecteurs-écrivants. À la croisée des pôles des usagers et des rédacteurs, la rubrique du courrier des lecteurs se présente avant tout comme le fruit de cette interaction.

\title{
Conclusion
}

Au terme de ce bref parcours synthétique sur la rubrique du courrier des lecteurs, quelles conclusions pouvons-nous tirer ? Et, finalement, pouvons-nous parler d'une véritable co-construction entre usagers et médias par le biais de cette rubrique ? Il semble que le bilan doive être nuancé. Certes, à travers cette interface, se font jour les liens et interactions qui unissent les lecteurs à leur journal. La rubrique du courrier des lecteurs, relais médiatique du discours populaire, constitue également un accès privilégié à l'opinion des récepteurs. Leurs doutes, colères et espoirs y sont relayés mieux qu'ailleurs. En outre, les lecteurs, au-delà des simples vertus cathartiques reconnues aux rubriques de courrier, semblent aussi investir ces

1 La Nation Belge, le 03/12/1920, $\mathrm{n}^{\circ} 338, \mathrm{p} .1$.

2 Le Peuple, le $27 / 12 / 1980, \mathrm{n}^{\circ} 277$, p. 1. 
rubriques, et à travers elles leur journal, d'un rôle amplificateur et parfois même d'une mission particulière d'intercesseur auprès des personnes en charge de leur problème. À ce titre, elles présentent un réel intérêt dans l'étude du pôle des récepteurs. Toutefois, cette source connaît aussi ses limites, voulues par les lecteurs ou imposées par les journaux. D'une part, la rubrique du courrier des lecteurs reste avant tout aux mains d'une rédaction. Partie intégrante du journal, elle est gérée par lui. Aussi, les quotidiens et hebdomadaires effectuent-ils un inévitable tri au sein des courriers reçus et n'en publient qu'une partie, choisie par leurs soins et selon des critères qui leur sont propres. Tous les correspondants n'ont donc pas la certitude d'être publiés ou le sont parfois partiellement. En outre, les commentaires qu'ajoutent encore certains journaux canalisent également la lecture de ces courriers. La rubrique demeure donc un produit alimenté par les lecteurs-écrivants mais contrôlé par la presse.

D'autre part, au-delà de l'expression simple d'une opinion, on pourrait attendre de la rubrique qu'elle remplisse d'autres fonctions : l'énonciation de suggestions constructives, à même de faire évoluer des débats sociétaux, un véritable feedback renvoyé au journal, voire une participation active à l'élaboration de celui-ci. Ces missions, certes présentes, ne sont toutefois rencontrées que très partiellement dans les rubriques du courrier des lecteurs avant les années 1970. Sorte d'agora où se déposent les plaintes des uns et des autres, les rubriques se limitent bien souvent jusqu'alors à l'expression des misères de chacun. Dans les années 1970 cependant, les rubriques du courrier des lecteurs connaissent un véritable envol, impulsé par les rédactions. Les courriers abondent et s'insèrent au sein de rubriques régulières. Celles-ci s'ouvrent à de véritables débats où se multiplient progressivement les suggestions de changement au-delà des protestations autour de thématiques personnelles. Mais il serait sans doute abusif de parler alors d'une véritable co-construction entre les lecteurs et leur quotidien ou hebdomadaire. Il semble donc que, si les lecteurs ne participent guère activement à l'élaboration du journal par le biais de la rubrique du courrier des lecteurs, ils lui reconnaissent au moins un certain pouvoir d'action, comme intercesseur, mais surtout un rôle privilégié d'exutoire et de porte-voix.

Nous souhaiterions surtout terminer ces conclusions en soulignant l'intérêt que revêtirait une approche plus approfondie de ces moments où les usagers des médias ont été investis d'une autre fonction que celle de simples consommateurs capables de rétroactions 
et de réactions, aimablement consignées dans la rubrique du courrier. Plusieurs des articles rassemblés dans cette publication mettent en exergue des moments récents où pareille configuration a été rencontrée. Il serait pertinent de pouvoir poursuivre ce travail afin de déterminer dans quelle mesure ces rapports nouveaux entre usagers et producteurs de contenus médiatiques perdurent ou évoluent, et ne se limitent pas à une mise en œuvre opportuniste de simples stratégies marketing. Il nous paraît de même que serait digne d'être investiguée davantage la période de naissance de la presse où les rôles liés à la production du message ne semblaient pas encore définitivement arrêtés. Le bref survol par sondage de la situation vécue en Belgique réalisé ici se voulait une entrée en matière en ce sens. Le chantier d'une lecture transversale approfondie de l'ensemble des supports médiatiques jamais produits dans notre pays est désormais ouvert... 